\title{
Quantum mechanical balance equations for modeling transport in closed electric circuits.
}

\author{
Bart Sorée, Wim Magnus, Wim Schoenmaker \\ IMEC, Kapeldreef 75 \\ B-3001 Leuven, Belgium \\ Bart.Soree@imec.be,Wim.Magnus@imec.be,Wim.Schoenmaker@imec.be
}

\begin{abstract}
We derived a set of quantum mechanical balance equations for a closed electric circuit represented by a closed region $\Omega$. These equations were numerically simulated to obtain information about global and local transport properties. In particular we studied a pure quantum wire at low temperature containing only acoustical phonons. We also investigated the influence of a single elastic scatterer present in the quantum wire.
\end{abstract}

\section{Introduction}

In ref. [1], it was argued that the reservoir concept is not suitable to set up a quantum transport formalism. An alternative approach was proposed based on the non-trivial topology of closed electric circuits.

\section{Derivation of quantum mechanical balance equations}

The electric circuit is represented by a closed region $\Omega$ in which the electrons are confined. The region $\Omega$ also contains a battery that is responsible for the electromotive force (EMF) caused by an externally applied voltage. In general electromagnetic fields $(\vec{E}, \vec{B})$ will be present. The one-electron wave functions and the field operators $\hat{\Psi}$ vanish at the circuit boundary $\hat{\Psi}(\vec{r}, t)=0$ at $\partial \Omega$. The electromagnetic fields $\vec{E}$ and $\vec{B}$ are related to a scalar and vector potential $(\Phi, \vec{A})$. The total Hamiltonian can be written as $\hat{H}=\hat{H}_{e}+\hat{H}_{P}+\hat{H}^{\prime}$ where

$$
\hat{H}_{e}=\int_{\Omega} d \tau\left(\frac{1}{2} m \vec{V}^{*} \hat{\Psi}^{+} \cdot \vec{V} \hat{\Psi}+(U-e \Phi) \hat{\Psi}^{+} \hat{\Psi}\right) .
$$

Eq. (1) represents the electron energy in the presence of internal potentials $U$ and electromagnetic fields, where $\vec{V}=(-i \hbar \vec{\nabla}+e \vec{A}) / m$ is the velocity operator. $\hat{H}_{P}$ and $\hat{H}^{\prime}$ respectively represent the phonon bath energy and the electron-phonon interaction energy. The time evolution of operators in the Heisenberg picture is governed by the Heisenberg equation of motion $i \hbar \frac{\partial \hat{\Psi}}{\partial t}=[\hat{\Psi}, \hat{H}]$. This equation of motion is used to derive a set of local balance equations for energy and momentum. Integration of these local balance equations over the region $\Omega$ and taking the steady-state ensemble average then results in the following set of global balance equations in the steady-state regime :

$$
\begin{aligned}
I V_{\epsilon} & =\frac{i}{\hbar}\left\langle\left[\hat{H}_{e}, \hat{H}^{\prime}\right]\right\rangle=P\left(\Delta \mu, T_{e}\right) \\
\int_{\Omega} d \tau\langle\hat{\rho}\rangle \vec{E} & =\frac{i}{\hbar}\left\langle\left[\hat{\vec{P}}, \hat{H}^{\prime}\right]\right\rangle=\vec{F}\left(\Delta \mu, T_{e}\right)
\end{aligned}
$$


where $I V_{\epsilon}$ is the power delivered by the battery, while $\vec{F}\left(\Delta \mu, T_{e}\right)$ is the friction force arising from the phonons. The product $I V_{\epsilon}$ in Eq. (2) arises due to an integral theorem for closed electric circuits [2].

\section{Solving the balance equations}

In order to solve the balance equations (2)-(3) in the steady-state regime we use first order perturbation theory to calculate the ensemble average of an arbitrary Hermitian operator $\hat{A}(t)$ in the Heisenberg picture

$$
\langle\hat{A}(t)\rangle=\left\langle\hat{A}^{(0)}(t)\right\rangle_{0}-\frac{i}{\hbar} \int_{0}^{t} d t^{\prime}\left\langle\left[\hat{A}^{(0)}(t), \hat{H}_{i n t}^{(0)}\left(t^{\prime}\right)\right]\right\rangle_{0} .
$$

where the superscript $(0)$ denotes the interaction picture for operators. The total Hamiltonian is divided into an unperturbed part $\hat{H}_{0}$ and an interaction part $\hat{H}_{\text {int }}$. The initial density operator $\hat{\rho}_{0}$ is not chosen to be the Gibbs-ensemble. Instead, it is taken to be a "boosted" initial density operator, i.e.

$$
\begin{aligned}
\hat{\rho}_{0}^{B} & =\frac{1}{Z} \exp \left(-\beta_{e}\left(\hat{H}_{e}^{B}-\mu \hat{N}\right)\right) \exp \left(-\beta \hat{H}_{P}\right) \\
\hat{H}_{e}^{B} & =\hat{H}_{e}-\sum_{q} \hat{c}_{q}^{+} \hat{c}_{q} \frac{1}{2} \operatorname{sgn}\left(I_{q q}\right) \Delta \mu .
\end{aligned}
$$

The initial density operator $\hat{\rho}_{0}^{B}$ is parameterized by $\beta_{e}=1 / k_{B} T_{e}$ and $\Delta \mu$, where $I_{q q}$ is the current carried by the eigenstate $\mid q>$. Physically, it represents a moving and non-interacting electron system and a free phonon system in thermal equilibrium. The line integral of the electric field in the region $\Omega$ must be equal to the EMF, i.e.

$$
V_{\epsilon}=\oint_{C} \vec{E} \cdot d \vec{l}
$$

The independent variables $\Delta \mu, T_{e}$ and $V_{\epsilon}$ are found by solving Eqs. (2), (3) and (7) in a self-consistent way. A similar approach was also used in hot electron transport [3, 4].

\section{Results}

First, we have applied the balance equation approach to a pure quantum wire without impurities. The confinement is taken to be very strong and only sufficiently low temperatures are considered, so that only the lowest subband has to be taken into consideration. The dissipative medium consists of acoustical phonons only. The physical parameters that were taken for the quantum wire are listed in table 1 . In fig. 1 we show the results of a simulation where the current $I$ and the electron temperature $T_{e}$ are plotted versus the voltage $V_{\epsilon}$ ranging between $0.1 \mathrm{mV}$ and $1 \mathrm{~V}$. Apparently there exists a threshold of approximately $V_{\epsilon} \approx 0.1 \mathrm{~V}$ where the electron temperature $T_{e}$ starts to increase and where $I$ shows a non-linear behavior. Beyond this threshold the simulation results are to be doubted, because for higher electron temperature we need to take into account more than one subband and optical phonons in order to ensure energy relaxation. Next, we have studied the same quantum wire, but now containing one impurity. For the sake of simplicity we have modeled the impurity potential by a delta-function, i.e. $U(x)=U_{0} \delta(x)$. Accordingly, the electric field consists of a homogeneous part $E_{H}$ 


\begin{tabular}{||l|c|c||}
\hline Fermi energy & $\epsilon_{F}$ & $1 \mathrm{meV}$ \\
Lattice temperature & $T$ & $1 \mathrm{~K}$ \\
Effective mass & $m_{e}^{*}$ & $0.1 m_{e}$ \\
Lateral dimensions & $L_{y}, L_{z}$ & $10 \mathrm{~nm}$ \\
Wire length & $L_{x}$ & $1 \mathrm{~mm}$ \\
Sound velocity & $v_{s}$ & $10^{3} \mathrm{~m} / \mathrm{s}$ \\
Deformation potential & $D$ & $9 \mathrm{eV}$ \\
Mass density & $\rho_{0}$ & $10^{3} \mathrm{~kg} / \mathrm{m}^{3}$ \\
\hline
\end{tabular}

Table 1: Physical parameters
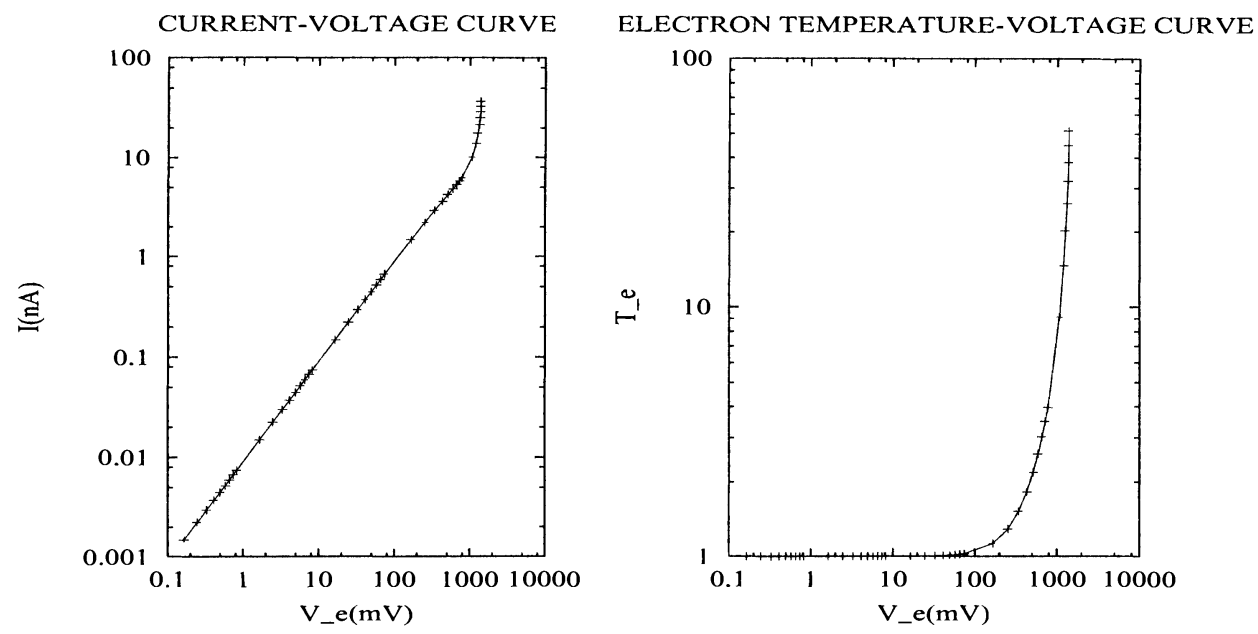

Fig. 1: Pure quantum wire

and a localized part $E_{\delta}(x)=V_{\delta} \delta(x)$. Due to Eq. (7) we have to require that the total voltage be equal to the sum of the homogeneous and localized voltage drop, i.e.

$$
\oint_{C} \vec{E} \cdot d \vec{l}=E_{H} L_{x}+V_{\delta}=V_{H}+V_{\delta}=V_{\epsilon}
$$

The results of the simulations are shown in fig. 2 where both the localized and the homogeneous voltage drop are shown as a function of the transmission coefficient $T\left(k_{F}\right)$ at Fermi-level. The total voltage drop is taken to be $1 \mathrm{mV}$. For a weak impurity potential, i.e. $T \approx 1.0$ we obtain the same results as the pure quantum wire. The total voltage drop $V_{\epsilon}$ tends to the homogeneous voltage drop $V_{H}$, while $V_{\delta} \rightarrow 0$. As the barrier height $U_{0}$ increases or the transmission coefficient $T$ becomes smaller than 1 , the homogeneous voltage drop decreases while the localized voltage drop increases. For a very large barrier height $U_{0}>>1$, or $T<<1.0$, the total voltage drop gets equal to the localized voltage drop $V_{\delta}$, while no voltage drop goes into the homogeneous part. If we look at the resistance for decreasing values of $T$ it seems that an "anomaly" occurs. Classically one expects a monotonic increase of the resistance when the barrier height increases. However, fig. 2 clearly shows that the resistance first decreases and subsequently increases for $T<0.1$. This phenomenon is due to the functional dependence 
of the transmission coefficient $T$ on the electron energy.
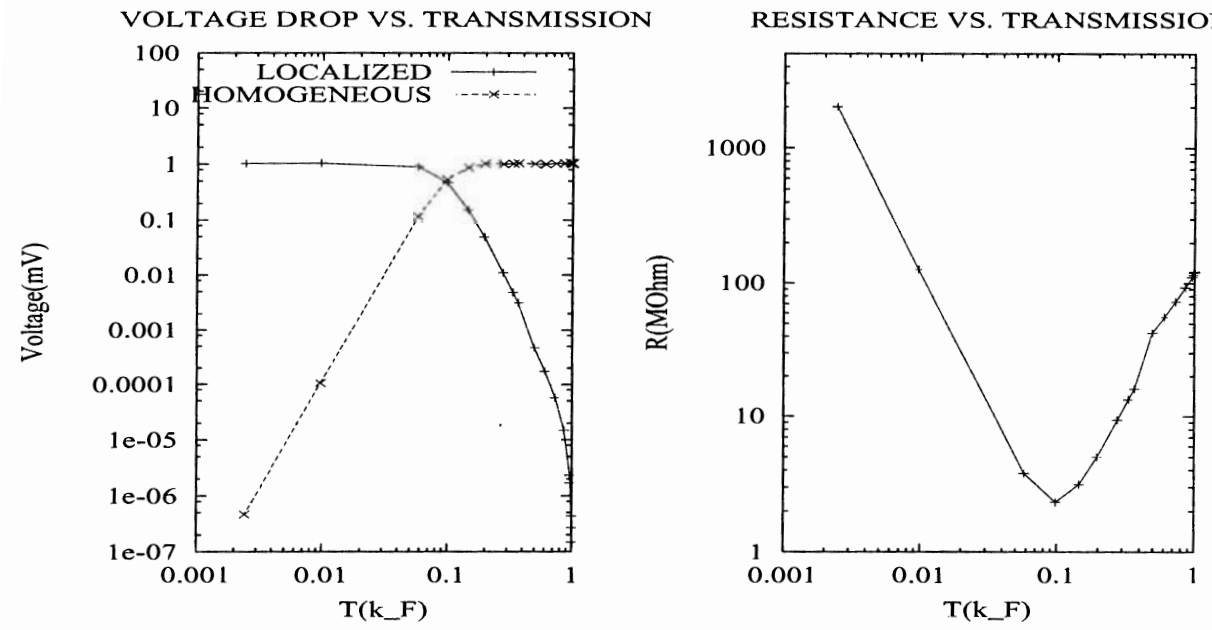

Fig. 2: Quantum wire with the presence of a delta-potential

\section{Conclusions}

The results we have obtained clearly show that the quantum mechanical balance equation approach is able to investigate global as well as local transport properties. In particular we demonstrated that the presence of an impurity in a quantum wire with strong confinement at low temperatures directly affects the spatial localization of energy dissipation. Moreover, this method resolves a longstanding problem of determining the spatial localization of energy dissipation in the presence of a potential barrier. In the future we will further apply the balance equation method to investigate spatially inhomogeneous structures such as quantum point contacts and carbon nanotubes.

Acknowledgment Part of this work is funded by the Flemish Institute for Science and Technology (IWT), Flanders (Belgium).

\section{References}

[1] W. Magnus and W. Schoenmaker. Quantized conductance, circuit topology and flux quantization. Phys. Rev. B, 61:10883, 2000.

[2] W. Magnus and W. Schoenmaker. On the use of a new integral theorem for the quantum mechanical treatment of electric circuits. J. Math. Phys., 39:6715, 1998.

[3] X.L. Lei and C.S. Ting A new approach to non-linear transport for an electronimpurity system in a static electric field. Journ. of Phys. C: Solid State Physics, 18:77-92, 1985.

[4] X.L. Lei and N.J.M. Horing Balance-equation approach to hot-carrier transport in semiconductors. Int. Journ. Mod. Phys. B, 6:801, 1992. 